\title{
ORIGINAL
}

\section{VARIABILIDAD, EFECTIVIDAD Y DESIGUALDAD. HISTERECTOMÍAS Y PROSTATECTOMÍAS POR ENFERMEDAD NEOPLÁSICA EN ESPAÑA (2002-2004)}

\author{
Salvador Peiró $(1,2)$ Ricard Meneu $(2,3)$ y Enrique Bernal-Delgado $(2,4)$ \\ Sanitat. Valencia. \\ (2) Grupo de Variaciones en la Práctica Médica en el Sistema Nacional de Salud. \\ (3) Fundación Instituto de Investigación en Servicios de Salud, Valencia. \\ (4) Instituto Aragonés de Ciencias de la Salud, Zaragoza.
}

(1) Centro Superior de Investigación en Salud Pública (CSISP). Dirección General de Salud Pública, Conselleria de

\section{RESUMEN}

Fundamento. Los debates sobre la equidad en la utilización de servicios sanitarios con frecuencia omiten considerar si las desigualdades observadas se producen en procedimientos efectivos o en tratamientos de efectividad no contrastada. El objetivo del estudio es ilustrar la diferencia entre desigualdad e inequidad desde la perspectiva de la investigación en servicios de salud.

Métodos. Estudio ecológico de las tasas estandarizadas de intervenciones quirúrgicas por cáncer de útero y próstata producidas entre 2002 y 2004 en 180 áreas sanitarias de España. Variables socioeconómicas: camas públicas por 1000 habitantes, nivel económico, tasa de paro (población 25-49 años), oficinas bancarias por 100.000 habitantes y proporción de personas analfabetas o sin estudios. Para estimar la desigualdad entre áreas se utilizaron estadísticos de Análisis de Área Pequeña. Para determinar el efecto de los factores socioeconómicos se utilizó ANOVA y regresión lineal Múltiple.

Resultados. Se analizaron 12.178 altas por cirugía oncológica de útero $(2,19$ por 10.000 mujeres) y 13.416 por cirugía en cáncer de próstata (2,47 por 10.000 hombres). Todos los estadísticos mostraron una mayor variabilidad en el cáncer de próstata. Las tasas de histerectomía no se relacionaron con ningún factor socioeconómico mientras que la prostatectomía se asoció a vivir en áreas con más camas ( $\beta=0,89, p<0,001)$, mayor nivel económico $(\beta=0,72, p=0,004)$ y menor tasa de analfabetos o sin estudios (según el tercil, Bt2=0,75, $\mathrm{p}=0,002 ; B \mathrm{t} 3=0,57, \mathrm{p}=0,044$ ).

Conclusiones. Las desigualdades en utilización de servicios no implican necesariamente inequidad. En el caso de la prostatectomía por cáncer de próstata, procedimiento de dudosa efectividad, las desigualdades observadas en contra de las áreas menos favorecidas no deberían interpretarse como prueba de inequidad.

Palabras clave: Variación. Equidad. Variación en la práctica clínica. Análisis de áreas pequeñas. Histerectomía. Prostatectomía.Tumores.

Correspondencia:

Salvador Peiró

Centro Superior de Investigación en Salud Pública (CSISP)

Avenida de Cataluña 21. 46020 Valencia

Correo electrónico: peiro_bor@gva.es
ABSTRACT

\section{Effectiveness, Variation and Inequalities. Histerectomies and Prostatectomies Due to Neoplasm in Spain (2002-2004)}

Background. Debates about equity in the utilization of health services often omit whether inequalities are observed in effective and safe procedures or they are experienced in treatments dubiously effective. This work tries to illustrate the difference between inequality and inequity in the health services research field.

Methods. Ecologic study on the standardized rates of surgical interventions in uterus and prostate cancer, produced between 2002 and 2004 in 180 healthcare areas in Spain. Socioeconomic variables: public beds per 100,000 inh, economic level, unemployment rate (population between 25 and 49), bank offices per 1,000 inh., and proportion of illiterate or persons with no studies. To estimate inequality statistics for Small Area Analysis were used; to determine the effect of socioeconomic factors, ANOVA and Linear Multiple Regression were modelled.

Results. 12,178 admissions for hysterectomy ( 2.19 per 10,000 women) and 13,416 prostatectomies ( 2.47 per 10,000 men) were analysed. All the statistics showed higher variation (inequality) in prostate cancer. Hysterectomy rates were not related with socioeconomic factors as oppose as prostatectomy: higher rates were related with living in areas with bigger centres $(\beta=0.89, \mathrm{p}<0.001)$, with more economic level $(\beta=0.72, p=0.004)$ and less rate of illiterate persons (with regard to the tertile, $\beta \mathrm{t} 2=0.75, \mathrm{p}=0,002 ; \mathrm{Bt} 3=0.57, \mathrm{p}=0,044$ ).

Conclusion. Inequalities in the utilization of healthcare services do not necessarily imply inequity. In prostatectomy due to prostate cancer, an uncertain procedure in terms of effectiveness, the observed inequalities against poorer areas, should not be interpreted as a symptom of inequity.

Keywords: Equity. Inequalities. Clinical practice variation. Small area analysis. Hysterectomy. Prostatectomy. Neoplasm.

Financiación: Este trabajo forma parte del Proyecto Atlas de Variaciones en la Práctica Médica en el Sistema Nacional de Salud que ha contado con ayudas de investigación del Instituto de Salud Carlos III (PI06/1673, PI05/2490, G03/202) y de la Obra Social y Cultural de Ibercaja.

Conflicto de intereses: Ninguno en relación con este manuscrito. 


\section{INTRODUCCIÓN}

La intervención del sector público en el ámbito sanitario en buena parte responde a argumentos igualitarios que buscan garantizar una atención médica cuya calidad sea independiente del lugar de residencia y de la capacidad económica de los individuos. Es lógico por tanto que un buen número de investigaciones se pregunten por el grado en que se alcanzan estos propósitos. La preocupación por las desigualdades en la utilización de servicios sanitarios y sus repercusiones sobre la equidad, un concepto más conflictivo, se ha traducido en una potente área de investigación. Mientras la constatación de "desigualdades" es una cuestión empírica, para afirmar que existe inequidad -una cuestión moral más compleja- se requiere disponer de suficiente información sobre las necesidades de las diferentes poblaciones, y realizar algunas asunciones (juicios de valor) sobre la definición concreta de necesidad, qué y hasta qué punto debe considerarse como tal ${ }^{1}$ y cuál es la adecuada respuesta a ésta. Las investigaciones acogidas al epígrafe de equidad en la prestación asistencia sanitaria se centran, sin embargo, en una de las posibles definiciones, de la equidad horizontal, la cual se define operativamente en términos de igual acceso a la asistencia o igual utilización por parte de individuos o grupos con similares necesidades.

De un modo sintético puede decirse que la cuestión de la equidad en la utilización de servicios de salud se ha venido analizando recurriendo a dos tipos de diseños y fuentes de información: 1) estudios basados en encuestas poblacionales, como las encuestas de salud y/o de presupuestos familiares, que contienen información específica sobre renta, educación, actividad laboral y otras variables socio-económicas, mientras que las variables relativas a la salud y la utilización sanitaria son muy genéricas; 2) estudios de pacientes $\mathrm{y}$ dolencias específicas, basados en registros administrativos, en los que las variables relativas a la enfermedad y la utilización están bastante bien recogidas pero no así las socioeconómicas.

Tanto la literatura sobre desigualdades como la literatura sobre variaciones en la práctica médica han constatado la ubicuidad e importancia de las diferencias (llámense desigualdades o variaciones) en utilización de servicios sanitarios, pero mientras en la primera se interpretan como expresión inequívoca de inequidad para la segunda son fundamentalmente un reflejo de la existencia de incertidumbre en la toma de decisiones clínicas, y su relación con la equidad es ambigua y debe ser interpretada con prudencia. Los estudios del primer tipo tienden a revelar que el Sistema Nacional de Salud (SNS) es, en conjunto, bastante equitativo en la provisión de servicios sanitarios, en contraste con los segundos, que suelen mostrar importantes desigualdades entre diferentes agregados poblacionales ${ }^{2}$. Algunos trabajos recientes apuntan que los buenos resultados obtenidos por los estudios de ámbito macro pueden estar enmascarando situaciones de vulneración de la equidad que se producen en el ámbito de colectivos específicos o diagnósticos y tratamientos concretos ${ }^{3}$.

A pesar de la contribución de estas investigaciones a la constatación de la gran heterogeneidad en la utilización de servicios sanitarios, algunos autores consideran que la literatura sobre variabilidad, por más iluminadora que resulte no mide directamente aspectos como la "accesibilidad" o la "equidad" de la asistencia, obviando que estos trabajos muestran la presencia constante, sistemática y abultada de "desigualdades" que pueden interpretarse como manifestación de "inequidad". Asumiendo la definición usual de equidad horizontal (igual tratamiento a igual necesidad médica, con 
independencia de otras características como renta, género, etnia, lugar de residencia, etc. $)^{4-6}$, los trabajos sobre variabilidad informan de importantes quiebras de dicho principio, dado que en ellos subyace una asunción de similitud de necesidades entre territorios acogida a la clásica noción bradshawniana de "necesidad comparativa"7. Con sus indudables limitaciones, la información aportada por las investigaciones sobre la variabilidad en la utilización de servicios sanitarios mediante el análisis de áreas pequeñas puede contribuir a superar algunas simplificaciones que, como mínimo, todavía confunden el marco conceptual y el desarrollo del estudio de las desigualdades.

Un aspecto esencial al analizar la relación entre la variabilidad poblacional en la utilización sanitaria y las diferencias socioeconómicas pasa por dilucidar si dichas asociaciones se presentan en todo tipo de procesos, o si se aprecian comportamientos distintos para diferentes condiciones clínicas. En este último caso, es importante conocer si los comportamientos detectados son consistentes con la "necesidad" y "efectividad" del servicio sanitario en estudio. Si las diferencias en utilización de servicios sanitarios muestran tendencias estables hacia la infrautilización de intervenciones efectivas (por ejemplo, la administración de aspirina en la prevención secundaria de la cardiopatía isquémica), podemos esperar problemas de equidad, casi con independencia de la definición que de ésta se adopte. Pero si las desigualdades en utilización se producen principalmente en tratamientos de efectividad no contrastada, las implicaciones sobre la equidad son más dudosas. Así, no es obvio que los territorios con menores tasas de cesáreas o de amigdalectomías estén recibiendo una atención inequitativa.

Avanzar en la interpretación de las desigualdades sanitarias y en sus implicacio- nes para la orientación de la política sanitaria, requiere analizar las relaciones entre utilización de servicios, su efectividad clínica y su variabilidad. Para valorar hasta qué punto diferentes desigualdades en utilización pueden suponer o no una quiebra de la equidad y, por tanto, para informar las políticas sanitarias, no basta con identificar desigualdades en utilización de servicios sanitarios, incluso si se establece que estas desigualdades se asocian a la renta o al nivel educativo, sino que es imprescindible valorar el tipo de intervención en el que se producen (efectivas o sujetas a incertidumbre sobre su efectividad), así como su adecuación a la situación clínica de los pacientes y la calidad técnica con que se realizan los procedimientos médicos implicados.

Este trabajo pretende ilustrar las diferentes implicaciones de las desigualdades en utilización de servicios según la efectividad de los procedimientos estudiados usando como ejemplo las intervenciones quirúrgicas en el cáncer de útero (un proceso en el que existe un claro acuerdo sobre la superioridad de la cirugía frente a otras alternativas de tratamiento) y el de próstata (un proceso en el que la indicación quirúrgica es muy controvertida). $\mathrm{La}$ selección de ambos tipos de procedimientos se realizó bajo la asunción de que el manejo quirúrgico en el cáncer de útero supone un estilo de práctica acorde con la evidencia científica $y$, en general, se corresponde con un servicio efectivo realizado en función de las necesidades de las pacientes, mientras la cirugía en el cáncer de próstata sería un procedimiento de efectividad cuestionada y no siempre acorde con la necesidad (en el anexo 1 se describen las características de ambos procedimientos que fundamentan estas asunciones). Asumiendo que, una vez controlado el efecto de la edad, la prevalencia de enfermedad es similar entre las diferentes áreas de salud, la variabilidad en la cirugía del cáncer de útero implica- 
ría la existencia de desigualdades y su asociación inversa a variables socio-económicas sugeriría fuertemente problemas de inequidad en el SNS. Por el contrario, las variaciones en intervenciones de cáncer de próstata y su asociación a variables socio-económicas, aun suponiendo desigualdades, sugerirían sobreutilización en las áreas con tasas elevadas, antes que un problema de inequidad.

El objetivo del estudio es analizar la variabilidad entre áreas de salud en las tasas de ambos tipos de intervenciones quirúrgicas y su posible asociación con variables socio-económicas

\section{MATERIAL Y MÉTODO}

Diseño. Estudio ecológico, descriptivo de las tasas estandarizadas de intervenciones quirúrgicas en cáncer de útero y cáncer de próstata, seguido de análisis de la variabilidad observada y de la asociación entre las tasas de intervenciones y determinados factores socioeconómicos.

Población/Ámbito. El objeto de análisis son las 180 Áreas de Salud de las 16 Comunidades Autónomas (CCAA) participantes en el Proyecto Atlas de Variaciones en la Práctica Médica en el Sistema Nacional de Salud (todas menos la Comunidad de Madrid, además de las ciudades autónomas de Ceuta y Melilla). Estas 180 áreas reunían una población empadronada de 36.664.474 habitantes (18.098.723 hombres y 18.565.751 mujeres) en promedio anual de los años 2002 a 2004.

Inclusión y exclusión de casos. De las altas incluidas en el Conjunto Mínimo de Datos Básicos (CMBD) de los años 2002 a 2004 de las 16 CCAA participantes se seleccionaron aquéllas con un diagnóstico principal de cáncer de útero o de próstata más un código de procedimiento quirúrgico relacionado con la extirpación de tejido ute- rino o prostático. En el anexo 2 se describen los códigos incluidos. Estos casos configuraron el numerador de las tasas de intervenciones oncológicas de útero y de próstata y fueron asignados al área de residencia del paciente, con independencia del hospital donde fueran operados. Los pacientes con residencia en el extranjero, Comunidad de Madrid, Ceuta o Melilla fueron excluidos. Los procedimientos utilizados para la asignación de altas al área de residencia han sido previamente descritos en otros trabajos $^{8,9}$.

Medidas de resultado. Como medida principal de resultado se utilizaron las tasas de intervenciones oncológicas de útero y próstata estandarizadas por edad (método directo $)^{8}$ respecto a las poblaciones de mujeres y hombres referidas en los padrones de 2002 a 2004.

Variables y definiciones. Se utilizaron las siguientes variables independientes:

- Camas hospitalarias de la red pública por 1.000 habitantes en cada área de salud (camas por $1.000 \mathrm{hab}$ ). Los datos se obtuvieron de la Encuesta de Establecimientos Sanitarios con Régimen de Internado correspondiente al año 2004 y para el denominador se utilizó el padrón de 2004.

- Nivel Económico. Es un índice de la renta familiar disponible por habitante (ingresos procedentes del trabajo, más las rentas de capital, prestaciones sociales y transferencias, menos los impuestos directos pagados por las familias y las cuotas pagadas a la seguridad social). Se estratifica en 10 categorías que se corresponden con determinados niveles de renta por habitante $(1:<7.200$ euros; 2 : 7.200 8.300; 3: 8.300-9.300; 4: 9.300-10.200; 5: 10.200-11.300; 6:11.300-12.100; 7:12.100-12.700; 8:12.700-13.500; 9: 13.500-14.500; y 10: >14.500). El dato, correspondiente al año 2003 se obtuvo por municipios del Anuario Económico 
de España 2005 y fue agregado por áreas de salud ponderando el nivel de cada municipio por su volumen de población.

- Tasa de paro en población de 25 a 49 años. El dato correspondiente al año 2004 se obtuvo por municipios del Anuario Económico de España 2006 y fue agregado por áreas en función del volumen de población de cada municipio.

- Oficinas bancarias por 100.000 habitantes (bancos por $100.000 \mathrm{hab}$ ). El dato, correspondiente al año 2004, se obtuvo por municipios del Anuario Económico de España 2006 y fue agregado del mismo modo que en los casos anteriores.

- Porcentaje de personas analfabetas y sin estudios en 2000. Corresponde a una variable censal, obtenida también del Anuario Económico de España 2004 y agregada por áreas. La variable se refiere a la "persona principal" de cada familia.

Análisis. En primer lugar, se calcularon las tasas crudas y estandarizadas de intervenciones oncológicas de útero y próstata, describiéndose éstas últimas mediante sus percentiles y un gráfico (dotplot) en el que la tasa estandarizada de cada área viene representada por un punto. A continuación se calcularon los estadísticos de variabilidad usuales en el análisis de áreas pequeñas ${ }^{8}$, utilizando salvo que se exprese lo contrario- las áreas situadas en los percentiles (P) 5 y 95 de la correspondiente distribución de tasas. Estos estadísticos fueron la razón de variación (RV5-95), razón de variación entre el área situada en el P75 y el P25 (RV25-75), Coeficiente de variación (CV5-95), Coeficiente de Variación Ponderado por el número de hombres o mujeres de cada área (CVP5-95), el Componente Sistemático de la Variación (CSV5-95), el Estadístico Empírico de Bayes (EB) y el Coeficiente de Gini. Las curvas de Lorenz asociadas a este coeficiente se representaron gráficamente para comparar la variabilidad entre los dos procesos estudiados.

Seguidamente se utilizó el análisis de la varianza de una vía (Anova Oneway) para valorar la capacidad explicativa del factor "comunidad autónoma" sobre la variabilidad en las tasas de intervenciones. Las tasas de las diferentes áreas de cada Comunidad se representaron mediante un gráfico de puntos cuyo volumen es proporcional al número de hombres o mujeres en cada área. Igualmente, se calcularon las razones estandarizadas de intervenciones (de forma similar a las razones estandarizadas de mortalidad) respecto al conjunto de las áreas estudiadas que se representaron mediante mapas ${ }^{8}$.

A continuación se realizó un análisis bivariable (Anova Oneway) para valorar las posibles asociaciones entre las tasas de las respectivas intervenciones y los factores socioeconómicos analizados. Las áreas de salud fueron previamente agrupadas en terciles para cada uno de los factores, siempre de menos a más privilegiadas (las tasas de paro y personas sin estudio se han ordenado de menos a más para mantener la misma dirección en todas las variables). Finalmente, se utilizó un modelo de regresión lineal múltiple para valorar las posibles asociaciones independientes entre estos factores y las tasas de cada uno de los procesos estudiados. Se utilizó el método por pasos (forward backward stepwise) con probabilidad de entrada de 0,05 y de salida de 0,10. Todos los análisis se realizaron utilizando el paquete estadístico STATA 10 (StataCorp, College Station, Texas)

\section{RESULTADOS}

Durante el período trascurrido entre los años 2002 a 2004 y en las 180 áreas de salud estudiadas se produjeron 12.178 altas por cirugía de cáncer de útero y 13.416 por cirugía de cáncer de próstata, con tasas crudas anualizadas de 2,19 y 2,47 intervenciones por 
cada 10.000 mujeres u hombres (tabla 1). En la figura 1 se muestra la distribución de las tasas de intervenciones en cada área. Respecto al cáncer de útero, el área en el P5 mostró

Tabla 1

Cirugía por cáncer de útero y próstata 2002-2004. Tasas anualizadas y estadísticos de variabilidad

\begin{tabular}{|c|c|c|c|}
\hline & & Útero & Próstata \\
\hline \multirow[t]{2}{*}{ Datos crudos } & Total intervenciones 2002-04 & 12.178 & 13.416 \\
\hline & Tasa cruda & 2,19 & 2,47 \\
\hline \multirow[t]{7}{*}{ Tasas estandarizadas por edad } & Tasa mínima & 0,00 & 0,26 \\
\hline & Tasa máxima & 4,77 & 8,61 \\
\hline & Percentil 5 & 1,16 & 0,60 \\
\hline & Percentil 25 & 1,87 & 1,15 \\
\hline & Percentil 50 & 2,19 & 2,00 \\
\hline & Percentil 75 & 2,55 & 3,03 \\
\hline & Percentil 95 & 3,07 & 4,72 \\
\hline \multirow[t]{7}{*}{ Estadísticos variabilidad } & Razón Variación $5-95$ & 2,61 & 7,72 \\
\hline & Razón Variación $25-75$ & 1,36 & 2,63 \\
\hline & Coeficiente Variación $_{5-95}$ & 0,19 & 0,51 \\
\hline & Coeficiente Variación Ponderado ${ }_{5-95}$ & 0,18 & 0,49 \\
\hline & Componente Sistemático Variación $_{5-95}$ & 0,01 & 0,19 \\
\hline & Estadístico Empírico de Bayes & 0,03 & 0,44 \\
\hline & Coeficiente de Gini & 0,14 & 0,34 \\
\hline \multirow{3}{*}{$\begin{array}{l}\text { Anova factor Comunidades } \\
\text { Autónomas }\end{array}$} & Coeficiente determinación $\left(\mathrm{r}^{2}\right)$ & 0,08 & 0,30 \\
\hline & Intervalo confianza $95 \% \mathrm{r}^{2}$ & $0,00-0,20$ & $0,07-0,53$ \\
\hline & $\mathrm{p}$ (Anova) & $\mathrm{p}=0,05$ & $\mathrm{p}<0,001$ \\
\hline
\end{tabular}

n=180 áreas de salud (de 16 Comunidades Autónomas; 36.664 .474 habitantes en promedio anual de 2002-2004). Todas las tasas están calculadas por 10.000 mujeres u hombres.

El subíndice indica que el correspondiente estadístico se ha calculado usando sólo las observaciones cuya tasa estandarizada en el correspondiente procedimiento estaba incluida entre los correspondientes percentiles.

Figura 1

Tasas de cirugía por cáncer de útero y próstata en las áreas de salud del Sistema

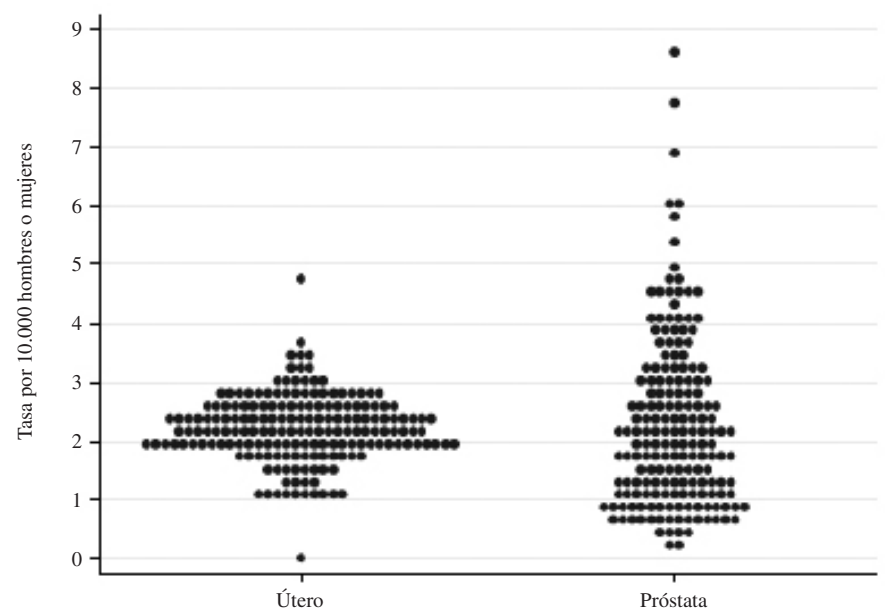

n=180 áreas de salud. Cada punto representa la tasa (estandarizada por edad y por 10.000 hombres o mujeres) en un área de salud. 
Figura 2

Curvas de Lorenz comparativas de la variabilidad en la distribución de las tasas de intervenciones oncológicas de útero y próstata

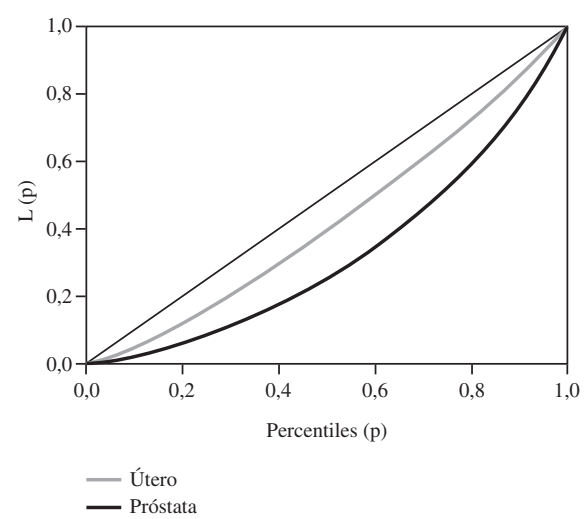

La curva representa las distribuciones acumuladas de áreas y tasas de intervenciones. una tasa estandarizada por edad de 1,16 intervenciones por 10.000 mujeres, mientras que el área en el P95 esta tasa fue de 3,07 intervenciones por 10.000 (2,6 veces mayor). En el cáncer de próstata la variación fue mucho mayor, con tasas estandarizadas por edad de 0,6 a 4,7 por 10.000 hombres y 7,7 veces más intervenciones en el área en el P95 respecto al área en el P5. Los estadísticos de variabilidad (tabla 1) confirman la mayor variación para las intervenciones por cáncer de próstata, como se refleja gráficamente en las curvas de Lorenz (figura 2).

El factor Comunidad Autónoma de pertenencia de las áreas explicó un $8 \%$ de la varianza en las tasas estandarizadas de intervenciones por cáncer de útero y un $30 \%$ en el caso de las de próstata (tabla 1). La figura 3 muestra las tasas de cada área agrupa-

Figura 3

Curvas de Lorenz comparativas de la variabilidad en la distribución de las tasas de intervenciones oncológicas de útero y próstata
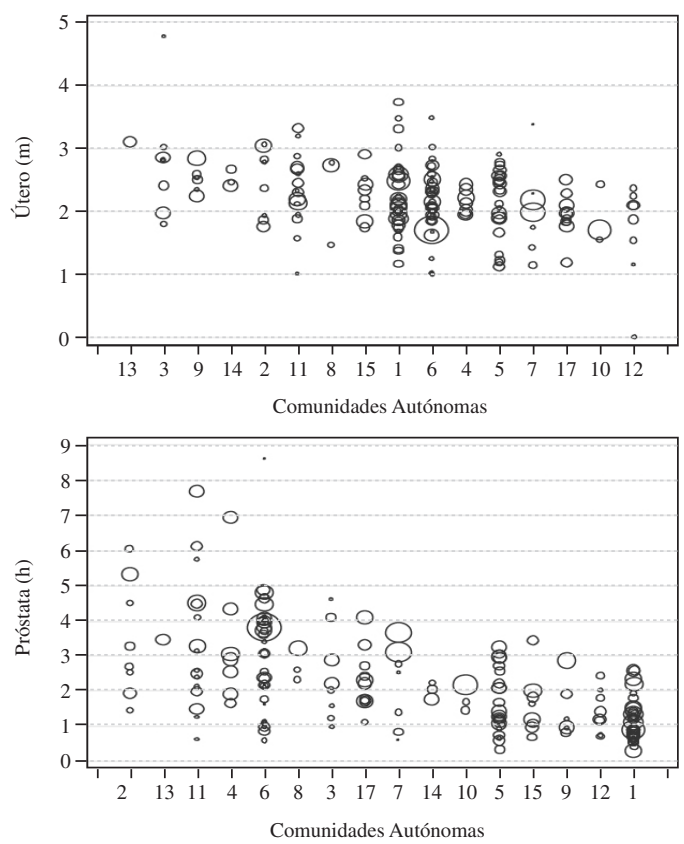

n=180 áreas y 16 Comunidades Autónomas. Cada punto representa la tasa (estandarizada por edad) de la respectiva intervención en un área, siendo su tamaño proporcional al número de hombres o mujeres en la respectiva área. Las Comunidades están ordenadas de mayor a menor tasa mediana en las respectivas intervenciones. 
Figura 4

Razones estandarizadas de intervenciones de cirugía por cáncer de útero y próstata por áreas de salud
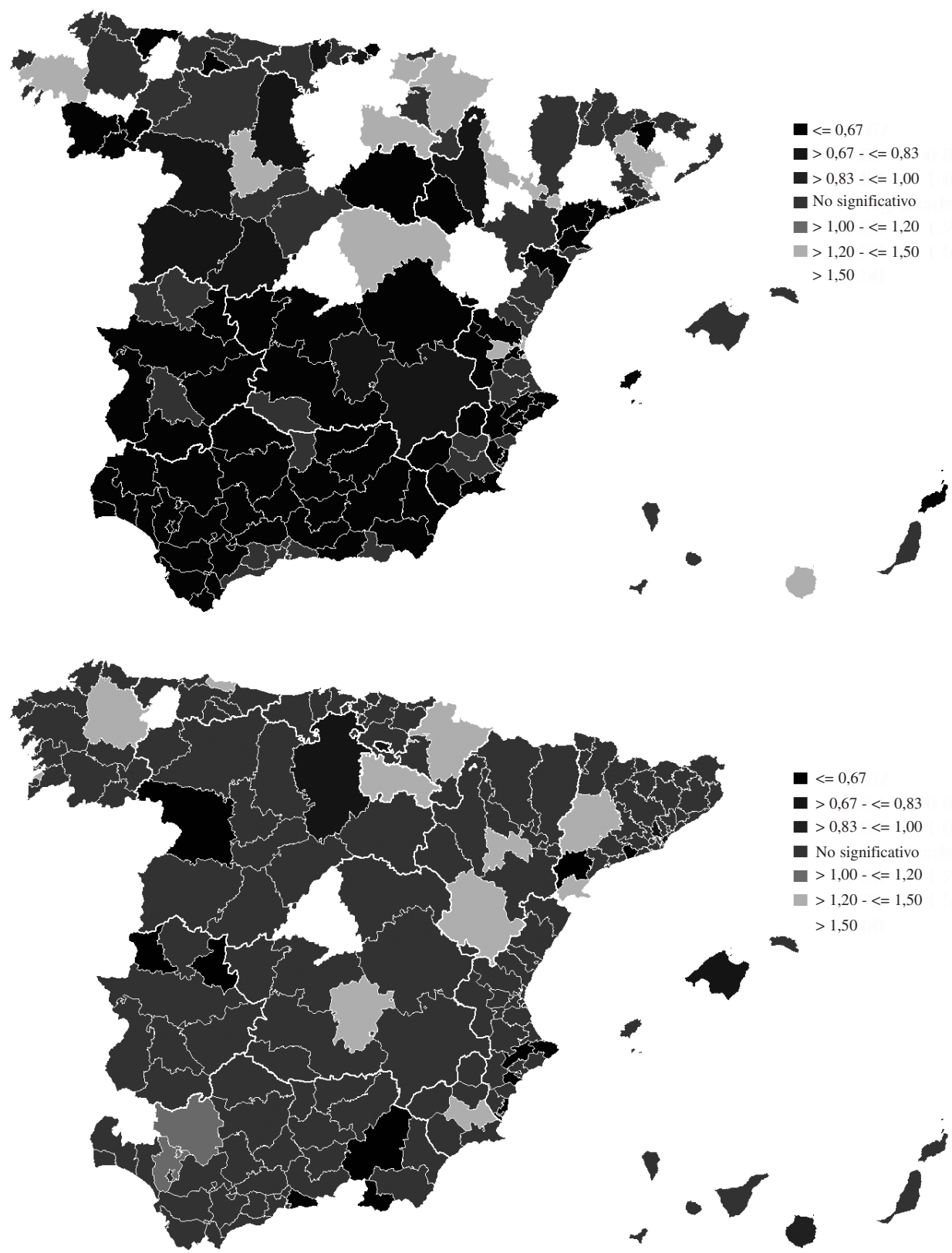

n=180 áreas. Las razones estandarizadas de intervenciones representan los casos observados en un área que respecto a los esperados para el conjunto de las mismas, estimados mediante el método indirecto. En blanco se representan las áreas sin datos. 
Figura 5

Correlación entre las tasas de intervenciones oncológicas por cáncer de útero y próstata en las áreas del Sistema Nacional de Salud

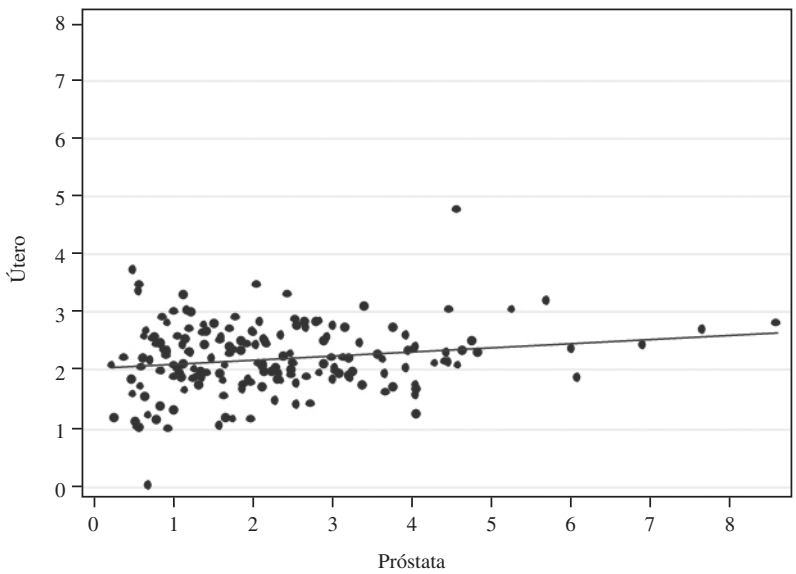

$\mathrm{n}=180$ áreas de salud. $\mathrm{r}^{2}=0,029 ; \mathrm{p}<0,021$.

das por CCAA (ponderadas por su tamaño poblacional y ordenadas según la tasa media de cada Comunidad). Se puede apreciar tanto el mayor gradiente entre CCAA en el caso de las intervenciones de próstata como la notable variabilidad entre las tasas de las áreas de las mismas CCAA. Los mapas de razones estandarizadas de intervenciones (figura 4) no parecen mostrar ningún patrón en el caso de la cirugía del cáncer de útero (de hecho, la mayor parte de las áreas no difieren significativamente de la media del SNS), mientras que la cirugía de cáncer de próstata muestra un patrón de tasas inferiores a la media en el sur de la península y superiores en el norte. La correlación entre las tasas de ambas intervenciones fue muy discreta $\left(r^{2}=0,03\right)$ (figura 5), aun siendo estadísticamente significativa $(\mathrm{p}=0,02)$.

En la tabla 2 se muestran las asociaciones bivariables entre diversos factores socioeconómicos y las tasas estandarizadas de cirugía de cáncer de útero y próstata. Las tasas de cirugía de útero no se asociaron a ninguna de las variables socioeconómicas estudiadas. En cambio, las tasas de cirugía por cáncer de próstata se asociaron positivamente al número de camas hospitalarias por 1.000 habitan- tes, mayor nivel económico, mayor número de oficinas bancarias por 100.000 habitantes, y negativamente a la tasa de paro en población de 25 a 49 años y al porcentaje de "persona principal de la familia" analfabetos o sin estudios. En algún caso, las diferencias en las tasas se producen entre las áreas situadas en los terciles primero y tercero, solapándose el tercil intermedio con alguno de los otros dos.

En el análisis multivariable las tasas de cirugía por cáncer de útero tampoco mostraron ninguna asociación con los factores socio-económicos analizados, no incorporándose ninguna variable a los modelos. Las tasas de cirugía por cáncer de próstata (tabla 3) mantuvieron la asociación con el número de camas hospitalarias por 1.000 habitantes (la tasa crece 0,89 puntos en el tercil superior respecto al primer tercil), el nivel económico (crecimiento de 0,72 puntos en el tercil superior respecto al primer tercil) y el porcentaje de "persona principal de la familia" analfabetos o sin estudios (crecimiento de 0,57 puntos entre el tercil con menor porcentaje de "sin estudios" respecto al tercil con mayor porcentaje de "sin estudios"). El número de oficinas bancarias y la tasa de paro no mantuvieron las asociaciones pre- 
Tabla 2

Asociación entre variables socioeconómicas y tasas de cirugía por cáncer de útero y próstata. Análisis bivariable

\begin{tabular}{|c|c|c|c|c|c|c|}
\hline & \multicolumn{2}{|c|}{ Terciles } & \multicolumn{2}{|c|}{ Útero } & \multicolumn{2}{|c|}{ Próstata } \\
\hline & $\mathbf{T}$ & Rango & Tasa [IC95\%] & $\mathbf{p}$ & Tasa [IC95\%] & $\mathbf{p}$ \\
\hline \multirow{3}{*}{$\begin{array}{l}\text { Camas hospital } \\
1.000 \text { hab. }\end{array}$} & $\mathrm{T} 1$ & $0,82-1,71$ & $2,09[1,95 ; 2,24]$ & \multirow[t]{3}{*}{0,182} & $1,93[1,65 ; 2,21]$ & \multirow[t]{3}{*}{$<0,001$} \\
\hline & $\mathrm{T} 2$ & $1,17-2,42$ & $2,29[2,14 ; 2,44]$ & & $1,86[1,58 ; 2,14]$ & \\
\hline & T3 & $2,42-6,15$ & $2,21[2,05 ; 2,36]$ & & $3,00[2,53 ; 3,47]$ & \\
\hline \multirow[t]{3}{*}{ Nivel económico } & $\mathrm{T} 1$ & $1,76-3,95$ & $2,17[2,00 ; 2,33]$ & \multirow[t]{3}{*}{0,440} & $1,63[1,31 ; 1,95]$ & \multirow[t]{3}{*}{$<0,001$} \\
\hline & $\mathrm{T} 2$ & $3,98-6,46$ & $2,28[2,11 ; 2,45]$ & & $2,11[1,79 ; 2,44]$ & \\
\hline & T3 & $6,47-9,40$ & $2,15[2,03 ; 2,27]$ & & $3,05[2,66 ; 3,44]$ & \\
\hline \multirow{3}{*}{$\begin{array}{l}\text { Tasa de paro } \\
25-49 \text { años }\end{array}$} & $\mathrm{T} 3$ & $8,02-14,25$ & $2,24[2,11 ; 2,37]$ & \multirow[t]{3}{*}{0,618} & $1,97[1,59 ; 2,35]$ & \multirow[t]{3}{*}{0,003} \\
\hline & $\mathrm{T} 2$ & $5,42-8,00$ & $2,14[1,96 ; 2,32]$ & & $2,03[1,75 ; 2,32]$ & \\
\hline & $\mathrm{T} 1$ & $1,20-5,36$ & $2,22[2,08 ; 2,35]$ & & $2,78[2,36 ; 3,20]$ & \\
\hline \multirow{3}{*}{$\begin{array}{l}\text { Bancos } \\
100.000 \text { hab. }\end{array}$} & $\mathrm{T} 1$ & $4,80-7,95$ & $2,17[2,03 ; 2,30]$ & \multirow[t]{3}{*}{0,486} & $1,79[1,49 ; 2,10]$ & \multirow[t]{3}{*}{0,008} \\
\hline & $\mathrm{T} 2$ & $8,04-9,36$ & $2,15[2,00 ; 2,30]$ & & $2,43[2,05 ; 2,81]$ & \\
\hline & $\mathrm{T} 3$ & $9,37-15,70$ & $2,27[2,10 ; 2,44]$ & & $2,56[2,15 ; 2,98]$ & \\
\hline \multirow[t]{3}{*}{$\%$ persona principal sin estudios } & $\mathrm{T} 3$ & $18,51-37,75$ & $2,30[2,11 ; 2,48]$ & \multirow[t]{3}{*}{0,254} & $1,52[1,23 ; 1,80]$ & \multirow[t]{3}{*}{$<0,001$} \\
\hline & $\mathrm{T} 2$ & $13,14-17,96$ & $2,16[2,03 ; 2,29]$ & & $2,49[2,16 ; 2,81]$ & \\
\hline & $\mathrm{T} 1$ & $4,70-12,96$ & $2,13[2,00 ; 2,27]$ & & $2,78[2,35 ; 3,21]$ & \\
\hline
\end{tabular}

T: Tercil; IC95\%: Intervalo de confianza del 95\%.

Tabla 3

Asociación entre variables socioeconómicas y tasas de cirugía por cáncer de próstata. Regresión lineal multivariante

\begin{tabular}{|l|c|c|c|}
\hline & Coef. & IC95\% & $\mathbf{p}$ \\
\hline Camas/1000 hab. (T3: 2,42-6,15) & 0,89 & 0,$48 ; 1,29$ & $<0,001$ \\
\hline \% Sin estudios (T2: 13,14-17,96) & 0,75 & 0,$28 ; 1,23$ & 0,002 \\
\hline \% Sin estudios (T3: 18,51-37,75) & 0,57 & 0,$01 ; 1,14$ & 0,044 \\
\hline Nivel económico (T3: 6,47-9,40) & 0,72 & 0,$23 ; 1,21$ & 0,004 \\
\hline Constante & 1.28 & $0.95 ; 1.62$ & $<0,001$ \\
\hline
\end{tabular}

$\mathrm{n}=180 ; \mathrm{r}^{2}=0,249 ; \mathrm{p}<0,0001$

T: Tercil; IC95\%: Intervalo de confianza del 95\%. Las variables relativas a oficinas bancarias y tasa de paro no fueron retenidas por el modelo.

viamente mostradas en el análisis bivariable al ser controladas por las otras variables incorporadas al modelo. En conjunto, las variables incorporadas explicaron un $24,9 \%$ de la varianza en las tasas estandarizadas de intervenciones por cáncer de próstata.

\section{DISCUSIÓN}

Los resultados de este estudio confirman las hipótesis previas y muestran que la cirugía oncológica de útero no presenta desigualdades territoriales de utilización relevantes y que, en todo caso, éstas no se asocian a dispa- ridades en el nivel socioeconómico, aspecto que sugiere que no existen diferencias territoriales de accesibilidad a un procedimiento efectivo. Por su parte, la prostatectomía en cáncer de próstata se comportó como un procedimiento con grandes desigualdades territoriales y un gradiente de utilización asociado positivamente a las áreas socioeconomicamente más privilegiadas. No obstante, al tratarse de un procedimiento de dudosa efectividad (en los que más no siempre es mejor) puede cuestionarse la suposición de inequidad e incluso podría plantearse la hipótesis alternativa de que residir en áreas de menor renta sería un factor protector de resecciones de próstata innecesarias.

Los resultados de este trabajo son consistentes con un estudio previo sobre desigualdades en utilización de servicios hospitalarios para 9 procesos asistenciales seleccionados en función de su diversa efectividad ${ }^{10}$ y sugieren que aunque en el SNS existen importantes desigualdades entre áreas de salud éstas son menores en los procesos caracterizados por un elevado consenso clínico sobre su manejo, y más importantes en los procesos electivos o con mayor incertidum- 
bre sobre la efectividad de la indicación quirúrgica. Las desigualdades identificadas en el trabajo previo no mostraron una relación sistemática con las variables socioeconómicas o el sexo y sólo en algún caso, fundamentalmente en cirugía electiva o procesos con alta incertidumbre sobre su manejo, los factores socioeconómicos se asociaron a diferencias en los niveles de utilización. Adicionalmente hay que señalar que tanto este estudio como el trabajo previo coinciden en mostrar desigualdades de utilización de determinados servicios entre las diversas CCAA. Sin embargo, en ambos casos, la importancia de estas diferencias viene relativizada por la coexistencia de variaciones intra-comunidad autónoma de similar magnitud a las diferencias entre CCAA.

Mientras las desigualdades encontradas para la cirugía de útero son compatibles con posibles variaciones en la incidencia y el efecto del error aleatorio (sus estadísticos de variabilidad son similares a los de la hospitalización por fractura de cadera, ${ }^{11}$ proceso prototipo de baja variabilidad y debida exclusivamente a diferencias en incidencia), las amplias variaciones encontradas en la cirugía de cáncer de próstata -más de un $600 \%$ de diferencia entre las áreas en el P5 y P95- no son compatibles con diferencias entre las poblaciones estudiadas. Las causas de esta alta variación hay que buscarlas, previsiblemente, en las expectativas injustificadas sobre el cribado oportunista ${ }^{12-14}$, la incertidumbre sobre el mejor tratamiento en estadíos precoces que se transformaría en una mayor intensidad terapéutica en las áreas con más recursos, y la escasa tradición de toma de decisión compartida con los pacientes. Esta situación recomienda el abordaje de la incertidumbre mediante guías nacionales sobre diagnóstico y tratamiento de cáncer de próstata, así como adaptar a nuestro entorno algunos de los múltiples instrumentos ya disponibles para facilitar la incorporación de las preferencias de los pacientes a la toma de decisiones clínicas cuando no existe un único curso de acción claramente establecido ${ }^{15}$.
Entre las limitaciones de este trabajo hay que citar, en primer lugar, las inherentes a su diseño ecológico. El hecho de que no exista un gradiente socioeconómico en la tasas de intervenciones entre territorios es compatible con que los individuos más pobres en cada territorio tengan menor accesibilidad a los servicios sanitarios, un aspecto cuyo abordaje requiere diseños de base individual antes que ecológicos. En segundo lugar, el estudio ha utilizado ingresos en hospitales de utilización pública, excluyendo los del sector privado y es posible que la incorporación de las altas de estos centros dibujara un contorno más desigual del acceso a la atención, que afectaría más al conjunto del país que a la atención prestada por el SNS. En tercer lugar, hay que señalar que los resultados obtenidos en las dos patologías estudiadas no necesariamente son generalizables a otros procesos, y que probablemente puedan existir desigualdades en algunos procesos efectivos y distribuciones más igualitarias en algunos procesos sujetos a alta incertidumbre. Los factores explicativos de la variabilidad en la utilización de servicios sanitarios son muy diversos ${ }^{16,17}$ y pueden afectar en diferente forma a distintos procesos. Finalmente, en la interpretación de los resultados hay que tener en cuenta algunos problemas estadísticos (baja frecuencia, áreas con poblaciones muy diferentes) que pueden afectar a la precisión de las estimaciones. No obstante, los estadísticos que muestran un buen comportamiento en estas situaciones, como el CSV o el $\mathrm{EB}$, mantienen la variabilidad entre procedimientos consistente con el resto de parámetros.

Los resultados de este estudio tienen implicaciones de interés para las investigaciones sobre desigualdades pero también para la política y la gestión sanitaria. En el primer caso, recomiendan que los análisis sobre posibles desigualdades -territoriales, socioeconómicas u otras- en la utilización de servicios sanitarios deben tener en cuenta la diferente efectividad de las prestaciones existentes y, como norma, deberían evitarse las inferencias sobre posibles inequidades cuando se analizan condiciones sujetas a alta incertidumbre 
sobre su manejo terapéutico o en agregados de difícil interpretación como las "visitas al especialista" o "visitas al médico general. Por contra, el análisis de estas condiciones puede sugerir áreas de sobreutilización en las que podría mejorarse el uso de los recursos y, tal vez, reducir algunos riesgos asociados a las intervenciones sanitarias. En cuanto a las políticas prácticas, si el SNS pretende garantizar oportunidades similares de atención sanitaria a poblaciones similares, la distinta efectividad de las intervenciones supone una variable determinante a la hora de establecer las estrategias de mejora. Se trata de evitar que la obsesión por el "más es mejor" lleve a aumentar la utilización de intervenciones de beneficio dudoso, mientras no se realizan otras de efectividad demostrada y que, se quiera o no, compiten por los mismos recursos.

\section{BIBLIOGRAFÍA}

1. Goddard M, Smith P. Equity of access to health care services: theory and evidence. Soc Sci Med. 2001. 53: 1149-62.

2. Urbanos R, Meneu R. La investigación sobre desigualdades en utilización de servicios sanitarios y sus distintos abordajes. En: Rodríguez M, Urbanos R, eds. Desigualdades en salud. Factores determinantes y elementos para la acción. Barcelona: Elsevier-Masson; 2007. pp 145-65.

3. Urbanos Garrido RM. Equidad en el acceso a los servicios sanitarios: una revisión de la evidencia disponible. Inguruak Rev Sociol. 2007; 44:183-92.

4. No authors listed. Measuring inequity in health service delivery. Quantitative Techniques for Health Equity Analysis. Technical Note 13. Washington: The World Bank Group; 2005. Accesible en: http://siteresources.worldbank.org

5. Wagstaff A, van Doorslaer E, Paci P. On the measurement of horizontal inequity in the delivery of health care. J Health Econ. 1991; 10:169-205.

6. Wagstaff A, van Doorslaer E. Measuring and testing for inequity in the delivery of health care. J Human Resources. 2000; 35:716-33.

7. Bradshaw J. A taxonomy of social need. In: Mclachlan G, ed. Problems and progress in medical care. Oxford: Nuffield Provincial Hospital Trust; 1972.
8. Librero J, Rivas F, Peiró S, Allepuz A, Montes Y, Bernal-Delgado E, et al. Metodología del Atlas de variaciones en cirugía ortopédica y traumatología en el Sistema Nacional de Salud. Atlas Var Pract Med Sist Nac Salud. 2005; 1:43-8.

9. Oterino de la Fuente D, Castaño E, Librero J, Peiró S, Bernal-Delgado E, Martínez N, et al. Variaciones en hospitalizaciones pediátricas: métodos. Atlas Var Pract Med Sist Nac Salud. 2006; 2: 129-32.

10. Meneu R, Peiró S. Efectividad de los tratamientos y desigualdades en utilización de servicios sanitarios. En: Rodríguez M, Urbanos R, eds. Desigualdades en salud. Factores determinantes y elementos para la acción. Barcelona: Elsevier-Masson; 2007. pp 168-87.

11. Grupo de Variaciones en la Práctica Médica de la Red temática de Investigación en Resultados y Servicios de Salud (Grupo VPM-IRYS). Variaciones en cirugía ortopédica y traumatología en el Sistema Nacional de Salud. Atlas Var Pract Med Sist Nac Salud. 2005; 1:17-36.

12. Pashayan N, Powles J, Brown C, Duffy SW. Excess cases of prostate cancer and estimated overdiagnosis associated with PSA testing in East Anglia. Br J Cancer. 2006; 95: 401-5.

13. Lu-Yao G, Albertsen PC, Stanford JL, Stukel TA, Walker-Corkery ES, Barry MJ. Natural experiment examining impact of aggressive screening and treatment on prostate cancer mortality in two fixed cohorts from Seattle area and Connecticut. BMJ. 2002; 325: 740.

14. Etzioni R, Penson DR, Legler JM, di Tommaso D, Boer R, Gann PH, et al. Overdiagnosis due to prostate-specific antigen screening: lessons from US prostate cancer incidence trends. J Natl Cancer Inst 2002; 94:981-90.

15. Meneu R. Comunicación médico-paciente. Participación de los pacientes en las decisiones asistenciales. En: Aranaz JM, Aibar C, Vitaller J, Mira JJ, eds. Gestión Sanitaria. Calidad y Seguridad de los Pacientes. Madrid: Fundación MAPFRE, Ediciones Díaz de Santos; 2008.

16. Meneu R. Variabilidad de las decisiones médicas y su repercusión sobre las poblaciones. Barcelona: Masson; 2002.

17. Peiro S, Bernal-Delgado E. ¿A que incentivos responde la utilización hospitalaria en el Sistema Nacional de Salud? Gac Sanit. 2006; 20(Suppl 1):110-6. 
Anexo 1

\section{Efectividad de las intervenciones quirúrgicas por cáncer de útero y próstata}

La localización tumoral más frecuente de cáncer de útero es la de cuerpo uterino, y su tipo histológico más habitual el adenocarcinoma de endometrio. El cáncer de cérvix, con menor tasa de incidencia pero cifras de mortalidad ajustada por edad similares al anterior, suele originarse sobre lesiones precursoras y es más frecuente en mujeres de bajo nivel socioeconómico. El pronóstico de estos cánceres es relativamente bueno, con supervivencias relativas ajustadas por edad a los cinco años en España del 73,6\% para neoplasias del cuerpo del útero y del 60,4\% para tumores de cérvix.

El tratamiento recomendado para el cáncer de endometrio con enfermedad localizada es la cirugía, típicamente la histerectomía abdominal total, salpingo-ooforectomía bilateral y disección de ganglios linfáticos pélvicos y paraaórticos mediante un abordaje abdominal. En el resto de estadios debería añadirse radioterapia. La necesidad de linfadenectomía pélvica y aórtica en todas las pacientes con enfermedad limitada al útero es un tema controvertido. La vía quirúrgica tradicional y más habitual es la abdominal, aunque con equipos expertos podría realizarse la vía vaginal asistida por laparoscopia. El tratamiento de la recidiva puede abordarse mediante cirugía, radioterapia, hormonoterapia y quimioterapia. El tratamiento de elección en los sarcomas de útero es la cirugía, de forma parecida al cáncer de endometrio.

Para el cáncer de cérvix, se recomienda histerectomía radical (extirpación del útero con un manguito vaginal y con los tejidos de los parametrios y paracolpos) en enfermedad FIGO IB1 si no hay contraindicación de cirugía. La linfadenectomía pélvica también forma parte de la intervención quirúrgica. En cambio, la extirpación anexial no es un componente de la histerectomía radical. La extensión de la radicalidad quirúrgica depende del tamaño del tumor y de si existen signos de extensión hacia el parametrio o hacia la vagina.

La intervención quirúrgica del cáncer de próstata suscita una importante controversia. El abordaje terapéutico del cáncer de próstata localizado se centra en la vigilancia expectante, no debiendo someterse al paciente de forma rutinaria a terapias radicales. En el caso de riesgo intermedio no existen pruebas suficientes a favor de la estrategia quirúrgica o la vigilancia. En el caso del cáncer de alto riesgo se considera que la radioterapia radical o la cirugía radical son las mejores estrategias, aunque la decisión debería ser tomada en función de los valores individuales del paciente y su esperanza de vida. En el cáncer de próstata localmente avanzado se recomienda utilizar radioterapia. En tumores T3a-T4, se recomienda acompañada de hormonoterapia, como terapia neoadyuvante hormonal y adyuvante si el Gleason es mayor de 8. La radioterapia pelviana podría ser considerada en pacientes con tratamiento radioterápico que reciben hormonoterapia neoadyuvante y tienen participación de ganglios linfáticos pelvianos. En el caso de la enfermedad metastásica el tratamiento de primera línea son los agonistas LHRH o la orquidectomía bilateral. En los casos refractarios a hormonoterapia, las terapias alternativas son la quimioterapia o la terapia con dexametasona.

En síntesis, salvo para el tumor localizado en estadio de alto riesgo (PSA>20 ng/ml y Gleason >8) la cirugía no se considera un tratamiento superior a otras opciones, y en la mayor parte de los casos la supervivencia es buena y puede optarse por la vigilancia expectante (especialmente si se desea evitar la impotencia, una frecuente complicación de la cirugía).

Anexo 2

Selección de casos

\begin{tabular}{|c|c|c|c|c|}
\hline & Códigos & CIE9MC como diagnóstico principal & Códigos & CIE9MC de procedimiento \\
\hline \multirow[t]{7}{*}{ Cirugía Cáncer de útero } & 179 & Neo maligna útero parte sin especificar & 68.3 & Histerectomía subtotal abdominal \\
\hline & 180.x & Neo maligna de cérvix & 68.4 & Histerectomía abdominal total \\
\hline & 182.x & Neo maligna cuerpo uterino & 68.51 & Histerectomía vaginal laparoscópica \\
\hline & 233.1 & Carcinoma cérvix uterino in situ & 68.59 & Otra histerectomía vaginal \\
\hline & 233.2 & Carcinoma in situ de otras partes del útero & 68.6 & Histerectomía abdominal radical \\
\hline & & & 68.7 & Histerectomía vaginal radical \\
\hline & & & 68.8 & Exenteración pélvica \\
\hline \multirow[t]{8}{*}{ Cirugía Cáncer de próstata } & 185 & Neo maligna de próstata & 60.21 & Prostatectomía transuretral guiada láser \\
\hline & 233.4 & Carcinoma in situ de próstata & 60.29 & Otra prostatectomía transuretral \\
\hline & 236.5 & Neo próstata comportamiento no determinado & 60.3 & Prostatectomía suprapúbica \\
\hline & & & 60.4 & Prostatectomía retropúbica \\
\hline & & & 60.5 & Prostatectomía radical \\
\hline & & & 60.61 & Escisión local lesión próstata \\
\hline & & & 60.62 & Prostatectomía perineal \\
\hline & & & 60.69 & Otra prostatectomía \\
\hline
\end{tabular}

La selección de casos requería la presencia combinada de al menos un código diagnóstico y uno de procedimiento. CIE9MC: Clasificación Internacional de Enfermedades 9 revisión Modificación Clínica. La x indica la selección de todos los posibles códigos con cualquier dígito/s siguientes. 
